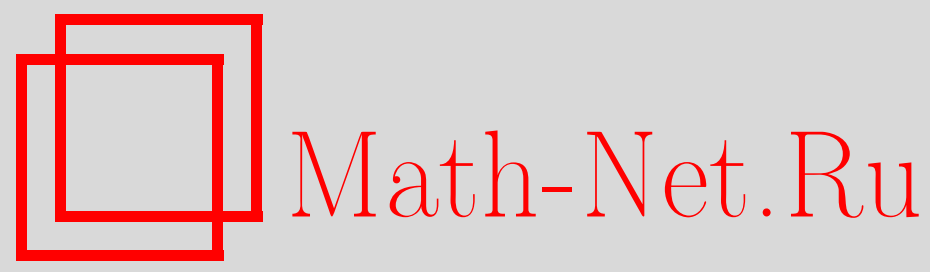

С. Я. Серовайский, Приближенное решение сингулярных оптимизационных задач, Матем. заметки, 2003, том 74, выпуск 5, 728-738

DOI: https://doi.org/10.4213/mzm305

Использование Общероссийского математического портала Math-Net.Ru подразумевает, что вы прочитали и согласны с пользовательским соглашением http://www . mathnet.ru/rus/agreement

Параметры загрузки:

IP : 3.80 .253 .173

26 апреля 2023 г., $16: 18: 48$

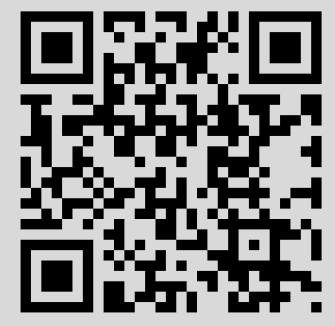




\title{
ПРИБЛИЖЕННОЕ РЕШЕНИЕ СИНГУЛЯРНЫХ ОПТИМИЗАЦИОННЫХ ЗАДАЧ
}

\author{
С. Я. Серовайский
}

Рассматривается задача оптимального управления для системы, описываемой нелинейным эллиптическим уравнением. Информация о существовании и единственности решения на конкретном управлении отсутствует. Допускается неразрешимость экстремальнй задачи. Осуществляется регуляризация задачи с помощью комбинации методов штрафа и Тихонова. Для регуляризованной задачи устанавливаются существование решения и необходимые условия оптимальности в форме вариационных неравенств. Показывается, что применяемый метод регуляризации обеспечивает нахождение в определенном смысле приближенного решения исходной задачи.

Библиография: 22 названия.

1. Постановка задачи. При исследовании оптимизационных задач можно столкнуться с различными сложностями. Возможно, на множестве допустимых управлений решение системы не существует или не единственно (см., например, [1]-[4]) или нарушение этих свойств возникает в процессе варьирования управления [5]. Экстремальная задача может оказаться неразрешимой (см., например, [6]-[9]) или некорректной в смысле Тихонова и Адамара [10], [11]. Трудности могут быть также обусловлены сложной структурой ограничений [12], вырождением условий оптимальности [13], негладкостью задачи (см., например, [9], [14], [15]). Анализ элементарных примеров оптимизационных задач с различньпи типами сингулярности проводится в [16]. Отличительная особенность рассматриваемой ниже задачи обусловлена наличием сразу двух (возможно, самых серьезных) сингулярностей - отсутствием однозначной разрешимости краевой задачи на любом управлении и возможной неразрешимостью оптимизационной задачи. Это обстоятельство требует модификации известных методов регуляризации (см., например, [1]-[4], [6]-[10]) и ослабления понятия приближенного решения экстремальной задачи.

В открытой ограниченной области $\Omega$ из $\mathbb{R}^{n}$ рассматривается система, описьваемая однородной задачей Дирихле для уравнения

$$
\Delta y+|y|^{\rho} y+u=0
$$

где $\rho>0, u$ - управление, $y$ - соответствующее ему состояние системы. Уравнение (1) при данном знаке перед нелинейным членом не имеет априорных оценок, что не позволяет применить для него известные методы исследования нелинейных уравнений эллиптического типа (см., например, [17]-[19]). Известно, что в этих условиях задача Дирихле 
для уравнения (1) может иметь не единственное решение [1]. Это обстоятельство еще не может служить препятствием для постановки и исследования оптимизационных задач для рассматриваемой системы [1], хотя сам факт неоднозначной разрешимости уравнений состояния не может не сказаться на особенностях используемых методов оптимизации.

Определим функционал

$$
I(u, y)=\frac{1}{r}\|y-z\|_{r}^{r}+\frac{\nu}{q}\|u\|_{q}^{q},
$$

где $z$ - известная функция класса $L_{r}(\Omega), \nu>0, r>1, q>1$, а через \|\|$_{p}$ обозначается норма в пространстве $L_{p}(\Omega)$. Задается вьпуклое замкнутое подмножество $U_{0}$ пространства $L_{q}(\Omega)$ и подпространство $Y$ функций класса $W_{s}^{2}(\Omega) \cap L_{r}(\Omega)$, обращающихся в нуль на границе $\Omega$, где $s=\min \{q, a\}, a=r /(\rho+1)$ при $r>\rho+1$ и $s=q$ при $r \leqslant \rho+1$. В соответствии с общей концепией исследования сингулярных управляемых систем [1] допустимой парой для уравнения (1) назовем такие функции $u \in U_{0}, y \in Y$, которые совместно удовлетворяют указанным соотношениям. Отметим, что мы не имеем здесь информации ни о существовании, ни о единственности решения краевой задачи на конкретном значении управления, хотя для некоторых управлений решение, безусловно, существует, причем, возможно, даже не единственное.

ЗАДАчА 1. Минимизировать функиионал I на множестве допустимых пар уравнения (1).

Исследование этой задачи осуществлялось Ж.-Л. Лионсом при $\rho=2, q=2, r=6$ (см. [1]). Подобные результаты, в принципе, можно получить и для других значений указанных параметров. Однако область их изменения остается весьма ограниченной и уж никак не выходит за рамки условий разрешимости оптимизационной задачи. Справедливо следующее утверждение.

ТЕОРема 1. При $\rho<r-1$ задача 1 разрешима.

ДокАЗАТЕЛЬСТВо. В силу ограниченности снизу функционала $I$ для задачи 1 существует минимизирующая последовательность, т.е. такая последовательность допустимых пар $\left\{\nu_{k}\right\}=\left\{u_{k}, y_{k}\right\}$, что справедлива сходимость

$$
I\left(\nu_{k}\right) \rightarrow \inf I
$$

где нижняя грань берется по множеству всех допустимых пар. В силу коэрцитивности функционала на произведении $L_{q}(\Omega) \times L_{r}(\Omega)$ из условия $(2)$ следует ограниченность на нем рассматриваемой последовательности. Поскольку пара $\left(u_{k}, y_{k}\right)$ является допустимой, ее компоненты связаны равенством $\Delta y_{k}+\left|y_{k}\right|^{\rho} y_{k}+u_{k}=0$. Тем самым функция $y_{k}$ удовлетворяет на $\Omega$ уравнению

$$
\Delta y_{k}=f_{k}
$$

с однородным граничньп условием, где $f_{k}=-\left|y_{k}\right|^{\rho} y_{k}-u_{k}$. При $\rho<r-1$ последовательность $\left\{\left|y_{k}\right|^{\rho} y_{k}\right\}$ ограничена в пространстве $L_{a}(\Omega)$, откуда следует ограниченность последовательности $\left\{f_{k}\right\}$ в классе $L_{s}(\Omega)$. Тогда, пользуясь классической теорией 
линейных уравнений эллиптического типа (см., например, [19]), установим ограниченность решений однородной задачи Дирихле для уравнения (3), т.е. последовательности $\left\{y_{k}\right\}$ в пространстве $W_{s}^{2}(\Omega)$, а значит, и в $Y$. После выделения подпоследовательности (с сохранением прежнего обозначения) имеем сходимость $u_{k} \rightarrow u$ слабо в $L_{q}(\Omega)$, $y_{k} \rightarrow y$ слабо в $Y$. Учитьвая компактность вложения $W_{s}^{2}(\Omega)$ в некоторое пространство $L_{p}(\Omega)$, после выделения подпоследовательности установим, что $y_{k} \rightarrow y$ сильно в $L_{p}(\Omega)$ и п.в. на $\Omega$. Имея сходимость $\left|y_{k}\right|^{\rho} y_{k} \rightarrow|y|^{\rho} y$ п.в. на $\Omega$ и ограниченность последовательности $\left\{\left|y_{k}\right|^{\rho} y_{k}\right\}$ в пространстве $L_{a}(\Omega)$, с помощью леммы 1.3 (см. [17, с. 25]) получаем $\left|y_{k}\right|^{\rho} y_{k} \rightarrow|y|^{\rho} y$ слабо в $L_{a}(\Omega)$. Переходя к пределу в уравнении (3), приходим к равенству (1). Учитьвая, что в силу слабой замкнутости множества $U_{0}$ справедливо включение $u \in U_{0}$, заключаем, что пара $\nu=(u, y)$ является допустимой. Пользуясь слабой полунепрерьвностью снизу степени от нормы, получим неравенство $\underline{\lim } I\left(\nu_{k}\right) \geqslant I(\nu)$, откуда в силу (2) следует, что пара $\nu$ оптимальна. Теорема доказана.

Теорема 1 является естественньм обобщением аналогичного результата, полученного Ж.-Л. Лионсом при $\rho=2, q=2, r=6$ (см. [1, с. 263]). Далее, в [1] проводилась регуляризация задачи с помощью метода адаптированного штрафа и устанавливались необходимые условия оптимальности для регуляризованной задачи. Эти результаты, по-видимому, остаются в силе и при $\rho<r-1$, т.е. для достаточно малых значений показателя нелинейности $\rho$ и больших значений показателя регулярности $r$ функционала по состоянию. Однако при доказательстве сходимости метода штрафа (переход к пределу в условиях оптимальности для регуляризованной задачи, см. [1, с. 268]) имеющейся регулярности рассматриваемых функций уже не достаточно для получения желаемых результатов. При больших значениях показателя нелинейности и низкой регулярности функционала мы не сможем воспроизвести приведенное выше доказательство в виду отсутствия информации об ограниченности последовательности $\left\{\left|y_{k}\right|{ }^{\rho} y_{k}\right\}$. Таким образом, в условиях большой степени нелинейности системы и относительно невысокой регулярности функционала приходится допустить возможность неразрешимости задачи 1 , вследствие чего математический аппарат, описанньй в [1], оказьвается вообще не пригодным.

Наша цель состоит в разработке алгоритма решения задачи 1 без каких-либо ограничений на параметры экстремальной задачи, в том числе, при ее возможной неразрешимости. Этот вариант не поддается анализу на основе стандартных методов оптимизации, ориентированных на получение необходимых условий оптимальности (см., например, [1], [3], [10], [20]), где разрешимость оптимизационной задачи служит непременным условием достижения желаемых результатов. Возможность анализа поставленной задачи без ограничений на ее параметры и при ее возможной неразрешимости обусловлена применением несколько иной формы метода регуляризации и ослаблением самого понятия приближенного решения задачи. На необходимость ослабления требований, предъявляемых к решению экстремальных задач, указывал еще Д. Гильберт. Впоследствии эта идея была реализована различными авторами (см., например, [6]-[10], [12], [21]).

2. Типы приближенных решений экстремальной задачи. С целью уточнения понятия приближенного решения рассмотрим абстрактную экстремальную задачу. Задаются линейные топологические пространства $V, W$, подмножество $V_{0}$ из $V$, оператор $A: V \rightarrow W$ и функционал $I$ на $V_{0}$. Требуется минимизировать функционал $I$ на 
множестве $U=\left\{\nu \in V_{0} \mid A \nu=0\right\}$. Для задачи 1 в качестве $V$ выбирается произведение пространств $L_{q}(\Omega) \times L_{r}(\Omega)$ со слабой топологией, множество $V_{0}$ равно $U_{0} \times Y$, а оператор $A$, определенный на произведении $L_{q}(\Omega) \times Y$, характеризуется равенством $A(u, y)=\Delta y+|y|^{\rho} y+u$. Тогда допустимые пары уравнения (1) описьваются соответствующим множеством $U$, а задача 1 сводится к указанной абстрактной форме.

В случае ограниченности снизу функционала $I$ существует его нижняя грань на множестве $U$. Тогда найдется такая последовательность $\left\{\nu_{k}\right\}$ из $U$, что имеет место сходимость $I\left(\nu_{k}\right) \rightarrow \inf I(U)$. Зная минимизирующую последовательность, в качестве приближенного решения задачи можно выбрать ее элемент с достаточно большим номером. Тем самьп для любого $\delta>0$ определяется такое значение $\nu^{*} \in U$, что справедливо неравенство $I\left(\nu^{*}\right) \leqslant \inf I(U)+\delta$. Приближенное решение $\nu^{*}$ есть такое допустимое значение, функционал на котором сколь угодно близок к его нижней грани. Точное решение задачи может при этом не существовать, а в случае его существования близость к нему значения $\nu^{*}$ в топологии пространства $V$ не гарантирована.

В теории оптимального управления выделяются задачи, корректные в смысле Тихонова [10], которые заведомо разрешимы, а любая минимизирующая последовательность сходится к их решению. С помощюю такой последовательности можно обеспечить не только близость соответствующего функционала к его нижней грани, но и близость самого выбранного приближения к решению задачи $\nu_{0}$ в естественной топологии пространства управлений. Тогда для любого $\delta>0$ и любой окрестности $O\left(\nu_{0}\right)$ точки $\nu_{0}$ может быть определено такое значение $\nu^{*} \in U$, что справедливы включение $\nu^{*} \in O\left(\nu_{0}\right)$ и неравенство $I\left(\nu^{*}\right) \leqslant \inf I(U)+\delta$. Условие минимизируемости последовательности и последнее соотношение можно также записать в виде неравенств $I\left(\nu_{0}\right) \leqslant \inf \lim I\left(\nu_{k}\right) \leqslant \inf I(U)$ и $I\left(\nu_{0}\right) \leqslant I\left(\nu^{*}\right) \leqslant \inf I(U)+\delta$.

Отметим, что построение минимизирующих последовательностей, наверняка сходящихся к оптимальному управлению, оказьвается чрезвычайно сложной проблемой. В этой связи представляется целесообразным ослабить требования, предъявляемыек приближенному решению задачи. Раз уж допускается минимизация функционала с какой-то погрешностью, представляется оправданным допустить, чтобы и равенство $A \nu=0$ вьполнялось приближенно. Под слабой минимизирующей последовательностью будем понимать такую последовательность $\left\{\nu_{k}\right\}$ из $V_{0}$, сходящуюся к решению $\nu_{0}$, что реализуется сходимость $A \nu_{k} \rightarrow 0$ в $W$ и условие $I\left(\nu_{0}\right) \leqslant \inf \lim I\left(\nu_{k}\right) \leqslant \inf I(U)$. Тогда можно дать новую форму приближенного решения задачи. Для любых $\delta>0$, окрестности $O\left(\nu_{0}\right)$ точки $\nu_{0}$ и окрестности $O(0)$ нуля в пространстве $W$ может быть определено такое значение $\nu^{*} \in\left(V_{0} \cap O\left(\nu_{0}\right)\right)$, называемое слабым приближенным $р$ шением задачи, что справедливо включение $A \nu^{*} \in O(0)$ и соотношение $I\left(\nu_{0}\right) \leqslant I\left(\nu^{*}\right)$ $\leqslant \inf I(U)+\delta$. Слабое приближенное решение есть значение, удовлетворяющее явным ограничениям на систему, а также данному уравнению с достаточной степенью точности, сколь угодно близкое в соответствующей топологии к решению задачи, с функционалом, сколь угодно близким к его нижней грани.

Хотя поиск слабого приближенного решения экстремальной задачи связан с сушественно меньшими трудностями по сравнению с обычньм приближенньм решением, эта проблема также может оказаться весьма сложной. Экстремальные задачи, как правило, не имеют решения, т.е. нижняя грань функционала на заданном множестве часто оказывается не достижимой. Отсутствие минимума функционала еще не делает задачу бессмысленной, поскольку его нижняя грань существует, а значит, может быть 
достигнута с определенной степенью точности. Отказьваясь от оптимальности значения $\nu_{0}$ в определении слабой минимизирующей последовательности и приближенного решения задачи, приходим к дальнейшему ослаблению этих понятий. Под ослабленной минимизирующей последовательностью будем понимать такую последовательность $\left\{\nu_{k}\right\}$ из $V_{0}$, что имеет место сходимость $\nu_{k} \rightarrow \nu_{0}$ в $V, A \nu_{k} \rightarrow 0$ в $W$ и неравенство $I\left(\nu_{0}\right) \leqslant \inf \lim I\left(\nu_{k}\right) \leqslant \inf I(U)$. Единственным отличием от предшествующего понятия здесь является отсутствие ограничений на $\nu_{0}$. В случае недостижимости нижней грани функционала оно уже не может быть решением задачи, что не мешает определить еще один класс приближенных решений экстремальной задачи. Под ослабленным приближенным решением задачи будем понимать значение $\nu^{*} \in V_{0}$, что для любых $\delta>0$, окрестности $O\left(\nu_{0}\right)$ некоторой точки $\nu_{0}$ пространства $V$ и окрестности $O(0)$ нуля в пространстве $W$ справедливы включения $A \nu^{*} \in O(0), \nu^{*} \in O\left(\nu_{0}\right)$ и неравенство $I\left(\nu_{0}\right) \leqslant I\left(\nu^{*}\right) \leqslant \inf I(U)+\delta$. Ослабленное приближенное решение есть значение, сколь угодно близкое (в указанном выше смысле) к множеству допустимых значений, обеспечивающее близость функционала в соответствующем смысле к его нижней грани. Очевидно, слабо приближенное решение задачи наверняка оказывается ослабленным приближенным.

В приведенных определениях отметим отсутствие каких-либо требований к точке $\nu_{0}$ из пространства $V$. Тогда ослабленная минимизирующая последовательность в пределе может дать значение функционала, не равное его нижней грани. Однако получаемое предельное значение функционала не может быть больше его нижней грани, в то время как справедливость уравнения состояния в пределе обеспечивается со все возрастающей степенью точности (значение оператора $A$ на нем стремится к нулю). Эти обстоятельства служат оправданием практического использования введенных понятий. Кроме того, существует класс задач, для которых любая ослабленная минимизирующая последовательность и приближенное решение совпадают с соответствующими слабыми понятиями. Так, в случае замкнутости множества $V_{0}$ в пространстве $V$ справедливо включение $\nu_{0} \in V_{0}$. Если оператор $A$ непрерьвен в точке $\nu_{0}$, то имеем сходимость $A \nu_{k} \rightarrow A \nu_{0}$ в $W$, а значит, $A \nu_{0}=0$. Тогда $\nu_{0}$ оказьвается допустимым управлением, а в силу неравенства $I\left(\nu_{0}\right) \leqslant \inf I(U)$ оно будет и оптимальным. Итак, в условиях замкнутости множества $V_{0}$ и непрерьвности оператора $A$ понятия слабого и ослабленного приближенных решений и соответствующие минимизирующие последовательности совпадают, а указанное управление $\nu_{0}$ будет решением задачи.

Ниже описьвается алгоритм, позволяющий строить ослабленные минимизирующие последовательности, а значит, находить ослабленные приближенные решения задачи 1 без каких-либо ограничений на ее параметры. В условиях существования решения задачи тот же алгоритм дает слабо минимизирующую последовательность, обеспечив в указанном вьше смысле и сколь угодно точное выполнение уравнения состояния, и сколь угодно высокую степень близости определяемого приближенного решения задачи к ее точному решению, и достижение нижней грани функционала с произвольной степенью точности. В качестве частного случая получаются известные результаты из монографии Ж.-Л. Лионса [1].

3. Регуляризованная оптимизационная задача. Рассмотрим задачу 1 в общей постановке, когда допускаются нарушение условия $\rho<r-1$ и неразрешимость задачи. Для регуляризации задачи вслед за Лионсом мы будем использовать метод штрафа, но не адаптированный, поскольку последний предполагает явное использование оптималь- 
ного управления. Отметим, что для последующего обоснования сходимости потребуются дополнительные априорные оценки, что, собственно, и не позволяет естественным образом распространить на общий случай известные результаты [1]. Для преодоления этих трудностей в минимизируемый функционал включается дополнительное слагаемое, в некотором смысле аналогичное стабилизатору в методе Тихонова [10].

Зададим вспомогательньй функционал

$$
I_{\varepsilon}(u, y)=I(u, y)+\frac{\varepsilon \beta}{b}\|y\|_{b}^{b}+\frac{1}{\varepsilon q}\|A(u, y)\|_{q}^{q},
$$

где $\varepsilon>0$ - параметр регуляризации, $b=\rho+1+\alpha, \alpha>0, \beta=0$ при $\rho<r-1$ и $\beta=1$ при $\rho \geqslant r-1$. В условиях теоремы существования решения задачи 1 регуляризованньй функционал соответствует стандартному методу штрафа [1], а при $\rho \geqslant r-1$ он включает в себя дополнительное слагаемое, стремящееся к нулю при $\varepsilon \rightarrow 0$. Поставим регуляризованную экстремальную задачу.

ЗАДАЧА 2. Минимизировать функиионал $I_{\varepsilon}$ на множестве $V_{0}$.

По аналогии с теоремой 1 установим следующее утверждение.

ТЕорема 2. Для любого $\varepsilon>0$ задача 2 разрешима.

ДокАЗАТЕЛЬСТво. В силу ограниченности снизу функционала $I_{\varepsilon}$ на множестве $V_{0}$ существует минимизирующая последовательность $\left\{u_{k}, y_{k}\right\}$ из $V_{0}$, т.е. справедливо условие $I_{\varepsilon}\left(u_{k}, y_{k}\right) \rightarrow \inf I_{\varepsilon}\left(V_{0}\right)$. Из определения $I_{\varepsilon}$ следует ограниченность последовательности $\left\{y_{k}\right\}$ в смысле $L_{c}(\Omega)$ и последовательностей $\left\{u_{k}\right\}$ и $\left\{g_{k}\right\}$ в $L_{q}(\Omega)$, где $g_{k}=\Delta y_{k}$ $+\left|y_{k}\right|^{\rho} y_{k}+u_{k}, c=r$ при $\rho<r-1$ и $c=b$ при $\rho \geqslant r-1$. Отсюда следует ограниченность в пространстве $L_{d}(\Omega)$ последовательности $\left\{f_{k}\right\}$, где $f_{k}=g_{k}-\left|y_{k}\right|^{\rho} y_{k}-u_{k}$, $d=\min \{q, c /(\rho+1)\}$. Функция $y_{k}$ оказьвается решением однородной задачи Дирихле для уравнения (3), а значит, она ограничена в пространстве $W_{d}^{2}(\Omega)$ (см. доказательство теоремы 1). После выделения подпоследовательностей, установим, что $u_{k} \rightarrow u$ слабо в $L_{q}(\Omega), y_{k} \rightarrow y$ слабо в $Z=W_{d}^{2}(\Omega) \cap L_{c}(\Omega)$. В результате получаем неравенство $\underline{\lim } I_{\varepsilon}\left(u_{k}, y_{k}\right) \geqslant I_{\varepsilon}(u, y)$, откуда следует, что пара $(u, y)$ является решением задачи 2 . Теорема доказана.

Безусловная разрешимость задачи 2 обеспечивается наличием дополнительной априорной оценки для элементов минимизирующей последовательности, получаемой с помощью второго слагаемого в определении регуляризованного функционала. Условия оптимальности для нее дает следующее утверждение.

ТЕОремА 3. Для того чтобь, пара $\nu_{\varepsilon}=\left(u_{\varepsilon}, y_{\varepsilon}\right)$ была решением задачи 2 необходимо, чтобы выполнялось вариачионное неравенство

$$
\int_{\Omega}\left(\nu\left|u_{\varepsilon}\right|^{q-2} u_{\varepsilon}+p_{\varepsilon}\right)\left(w+u_{\varepsilon}\right) d \Omega \geqslant 0 \quad \forall w \in U_{0} .
$$

При этом $p_{\varepsilon}$ и связанная с ней функиия $y_{\varepsilon}$ удовлетворяют однородным задачам Дирихле на $\Omega$ для уравнений

$$
\begin{gathered}
\Delta p_{\varepsilon}+(\rho+1)\left|y_{\varepsilon}\right|^{\rho} p_{\varepsilon}=-\left|y_{\varepsilon}-z\right|^{r-2}\left(y_{\varepsilon}-z\right)-\beta \varepsilon\left|y_{\varepsilon}\right|^{b-2} y_{\varepsilon} \\
\Delta y_{\varepsilon}+\left|y_{\varepsilon}\right|^{\rho} y_{\varepsilon}+u_{\varepsilon}=\varepsilon^{1 /(q-1)}\left|p_{\varepsilon}\right|^{q^{\prime}-1} p_{\varepsilon}
\end{gathered}
$$


ДоКАЗАТЕЛЬСТвО. Поскольку множество $V_{0}$ включает в себя ограничения на первый аргумент функционала $I_{\varepsilon}$ и не содержит ограничений на второй аргумент, необходимым условием экстремума для задачи 2 будет вариационное неравенство по первому аргументу и условие стационарности по второму аргументу [20]

$$
\begin{gathered}
I_{\varepsilon u}\left(\nu_{\varepsilon}\right)\left(w-u_{\varepsilon}\right) \geqslant 0 \quad \forall w \in U, \\
I_{\varepsilon y}\left(\nu_{\varepsilon}\right)=0,
\end{gathered}
$$

где $I_{\varepsilon u}$ и $I_{\varepsilon y}$ - частные производные функционала $I_{\varepsilon}$. Поскольку согласно теореме 2 оптимальное состояние $y_{\varepsilon}$ для задачи 2 оказьвается элементом пространства $Z$, под $I_{\varepsilon} y$ можно понимать соответствующую производную по подпространству $Z$ из $Y$. Это означает (см. [22]), что при варьировании функционала по второму аргументу приращение можно выбирать из класса $Z$.

Для любых функций $u, g$ из $L_{q}(\Omega), y$ из $Z$ и числа $\sigma$ справедливо равенство

$$
\begin{aligned}
I_{\varepsilon}(u+\sigma g, y)-I_{\varepsilon}(u, y)= & \frac{\nu}{q} \int_{\Omega}\left[|u+\sigma g|^{q}-|u|^{q}\right] d \Omega \\
& +\frac{1}{q \varepsilon} \int_{\Omega}\left[|A(u+\sigma g, y)|^{q}-|A(u, y)|^{q}\right] d \Omega \\
= & \sigma \nu \int_{\Omega}|u|^{q-2} u g d \Omega+\frac{\sigma}{\varepsilon} \int_{\Omega}\left[|A(u, y)|^{q-2} A(u, y) g\right] d \Omega+\eta(\sigma),
\end{aligned}
$$

где $\eta(\sigma) / \sigma \rightarrow 0$ при $\sigma \rightarrow 0$. В результате находим производную функционала по первому аргументу из условия

$$
I_{\varepsilon u}(u, y) g=\nu \int_{\Omega}|u|^{q-2} u g d \Omega+\frac{1}{\varepsilon} \int_{\Omega}\left[|A(u, y)|^{q-2} A(u, y) g\right] d \Omega \quad \forall g \in L_{q}(\Omega) .
$$

Подставляя это значение в неравенство (7) и полагая $p_{\varepsilon}=\varepsilon^{-1}\left|A\left(u_{\varepsilon}, y_{\varepsilon}\right)\right|^{q-2} A\left(u_{\varepsilon}, y_{\varepsilon}\right)$, установим соотношение (4). Учитьвая, что значение оператора $A$ на решении задачи 2 принадлежит пространству $L_{q}(\Omega)$, имеем включение $p_{\varepsilon} \in L_{q^{\prime}}(\Omega)$, где $1 / q+1 / q^{\prime}=1$. Из предшествующего равенства следует также справедливость условия (6).

Для любых функций $u$ пространства $L_{q}(\Omega), y, h$ из $Z$ и числа $\sigma$ имеем равенство

$$
\begin{aligned}
I_{\varepsilon}(u, y & +\sigma h)-I_{\varepsilon}(u, y)=\frac{1}{r} \int_{\Omega}\left[|y+\sigma h-z|^{r}-|y-z|^{r}\right] d \Omega \\
& +\frac{\varepsilon \beta}{b} \int_{\Omega}\left[|y+\sigma h|^{b}-|y|^{b}\right] d \Omega+\frac{1}{q \varepsilon} \int_{\Omega}\left[|A(u, y+\sigma h)|^{q}-|A(u, y)|^{q}\right] d \Omega \\
= & \sigma \int_{\Omega}|y-z|^{r-2}(y-z) h d \Omega+\sigma \varepsilon \beta \int_{\Omega}|y|^{b-2} y h d \Omega \\
& +\frac{\sigma}{\varepsilon} \int_{\Omega}\left\{|A(u, y)|^{q-2} A(u, y)\left[\Delta h+(\rho+1)|y|^{\rho} h\right]\right\} d \Omega+\eta(\sigma) .
\end{aligned}
$$

Отсюда следует соотношение

$$
\begin{aligned}
I_{\varepsilon y}(u, y) h= & \int_{\Omega}|y-z|^{r-2}(y-z) h d \Omega+\varepsilon \beta \int_{\Omega}|y|^{b-2} y h d \Omega \\
& +\frac{1}{\varepsilon} \int_{\Omega}\left\{|A(u, y)|^{q-2} A(u, y)\left[\Delta h+(\rho+1)|y|^{\rho} h\right]\right\} d \Omega \quad \forall h \in Z .
\end{aligned}
$$


В результате условие (8) принимает вид

$$
\int_{\Omega}\left\{\left[\left|y_{\varepsilon}-z\right|^{r-2}\left(y_{\varepsilon}-z\right)+\varepsilon \beta\left|y_{\varepsilon}\right|^{b-2} y_{\varepsilon}\right] h+p_{\varepsilon}\left[\Delta h+(\rho+1)\left|y_{\varepsilon}\right|^{\rho} h\right]\right\} d \Omega=0
$$

для любых функций $h$ из $Z$. Тогда функция $p_{\varepsilon}$ оказьвается решением задачи Дирихле для уравнения $(5)$ из класса $L_{q^{\prime}}(\Omega)$. Теорема доказана.

Результаты, аналогичные теоремам 2 и 3 , устанавливаются в [1] при $\rho=2, q=2$, $r=6$. Однако если обоснование разрешимости и условий оптимальности для регуляризованной задачи вьше в значительной степени соответствует математическому аппарату из [1] (за исключением производной по подпространству и вида регуляризованного функционала), исследование сходимости метода регуляризации потребует привлечения качественно иных приемов. Отсутствие ограничений на параметры приводит к существенным трудностям, практически не преодолимым в рамках известного подхода. В случае неразрешимости исходной задачи подобная техника вообе теряет смысл. Положительные результаты для общего случая получаются за счет использования понятия ослабленного приближенного решения.

4. Приближенное решение задачи. Для любого $\varepsilon>0$ задача 2 имеет решение $\nu_{\varepsilon}=\left(u_{\varepsilon}, y_{\varepsilon}\right)$, удовлетворяюшее соотношениям (4)-(6). Перейдем здесь к пределу при $\varepsilon \rightarrow 0$. Поскольку параметр $\varepsilon$ можно уменьшать дискретно, выражение $\left\{\nu_{\varepsilon}\right\}$ будем для простоты назьвать последовательностью. Справедливо следующее утверждение.

ТЕОРемА 4. Последовательность $\left\{\nu_{\varepsilon}\right\}$ является ослабленной минимизирующей для задачи 1.

ДокАЗАТЕЛьСТво. Поскольку функционал $I$ ограничен снизу на множестве $U$, здесь существует его нижняя грань. Тогда для всех $\delta>0$ найдется такое значение $\nu^{\delta}=$ $\left(u^{\delta}, y^{\delta}\right)$ из $U$, что справедливо неравенство $I\left(\nu^{\delta}\right) \leqslant \inf I(U)+\delta$. Учитьвая оптимальность пары $\nu_{\varepsilon}$ для задачи 2 , установим соотношение

$$
I_{\varepsilon}\left(\nu_{\varepsilon}\right)=\min I_{\varepsilon}\left(V_{0}\right) \leqslant I_{\varepsilon}\left(\nu^{\delta}\right)=I\left(\nu^{\delta}\right)+\frac{\beta \varepsilon}{b}\left\|y^{\delta}\right\|_{b}^{b} \leqslant \inf I(U)+\delta+\frac{\beta \varepsilon}{b}\left\|y^{\delta}\right\|_{b}^{b},
$$

откуда следует

$$
\varliminf_{\varepsilon \rightarrow 0} I_{\varepsilon}\left(\nu_{\varepsilon}\right) \leqslant \inf I(U)+\delta .
$$

Переходя к пределу при $\delta \rightarrow 0$, будем иметь

$$
\varliminf_{\varepsilon \rightarrow 0} I_{\varepsilon}\left(\nu_{\varepsilon}\right) \leqslant \inf I(U) .
$$

Пользуясь неравенством (9) и определением регуляризованного функционала, установим следующие оценки:

$$
\left\|y_{\varepsilon}\right\|_{r} \leqslant \text { const, } \quad\left\|u_{\varepsilon}\right\|_{q} \leqslant \text { const, } \quad\left\|A \nu_{\varepsilon}\right\|_{q} \leqslant \varepsilon^{1 / q} \text { const, }
$$

в правых частях которых находятся различные константы, не зависящие от $\varepsilon$. Тогда из $\left\{\nu_{\varepsilon}\right\}$ выделим такую подпоследовательность (сохраняя исходные обозначения), что имеет место сходимость $y_{\varepsilon} \rightarrow y_{0}$ слабо в $L_{r}(\Omega), u_{\varepsilon} \rightarrow u_{0}$ слабо в $L_{q}(\Omega), A \nu_{\varepsilon} \rightarrow 0$ слабо 
в $L_{q}(\Omega)$. Отсюда следует, что $\nu_{\varepsilon} \rightarrow \nu_{0}$ в $V$, где $\nu_{0}=\left(u_{0}, y_{0}\right)$. Учитьвая слабую полунепрерьвность снизу функционала $I$ в пространстве $L_{q}(\Omega) \times L_{r}(\Omega)$, получаем условие

$$
\varliminf_{\varepsilon \rightarrow 0} I_{\varepsilon}\left(\nu_{\varepsilon}\right) \geqslant I\left(\nu_{0}\right)
$$

Из определения регуляризованного функционала следует неравенство $I_{\varepsilon}(\nu) \geqslant I(\nu)$ для всех $\nu \in V$. Полагая $\nu=\nu_{\varepsilon}$ и переходя к пределу, установим

$$
\varliminf_{\varepsilon \rightarrow 0} I_{\varepsilon}\left(\nu_{\varepsilon}\right) \geqslant \underline{\lim }_{\varepsilon \rightarrow 0} I\left(\nu_{\varepsilon}\right) .
$$

Тогда, пользуясь условиями (9) и установленной сходимостью, будем иметь

$$
I\left(\nu_{0}\right) \leqslant \frac{\lim }{\varepsilon \rightarrow 0} I\left(\nu_{\varepsilon}\right) \leqslant \lim _{\varepsilon \rightarrow 0} I_{\varepsilon}\left(\nu_{\varepsilon}\right) \leqslant \inf I(U) .
$$

Из соотношений (11) и (12) следует, что последовательность $\left\{\nu_{\varepsilon}\right\}$ является ослабленной минимизирующей для задачи 3 . Теорема доказана.

При дополнительных условиях можно установить более сильньй результат.

ТЕОрема 5. При $\rho<r-1$ последовательность $\left\{\nu_{\varepsilon}\right\}$ является слабой минимизирующей для задачи 1.

ДокАЗАТЕЛЬСТво. Из последней оценки (10) следует, что функция $y_{\varepsilon}$ является решением однородной краевой задачи для уравнения

$$
-\Delta y_{\varepsilon}=\left|y_{\varepsilon}\right|^{\rho} y_{\varepsilon}+u_{\varepsilon}+f_{\varepsilon}
$$

причем для $f_{\varepsilon}$ имеет место сходимость $f_{\varepsilon} \rightarrow 0$ слабо в $L_{q}(\Omega)$. При вьполнении неравенства $\rho<r-1$ выражение в правой части уравнения (13) ограничено (см. доказательство теоремы 1$)$ в пространстве $L_{s}(\Omega)$. Тогда последовательность $\left\{y_{\varepsilon}\right\}$ ограничена в $W_{s}^{2}(\Omega)$, а значит, и в $Y$. Таким образом, имеет место сходимость $y_{\varepsilon} \rightarrow y_{0}$ слабо в $Y$. Повторяя рассуждения из доказательства теоремы 1 , установим, что $\left|y_{\varepsilon}\right|^{\rho} y_{\varepsilon} \rightarrow\left|y_{0}\right|^{\rho} y_{0}$ слабо в $L_{a}(\Omega)$. Переходя к пределу в равенствах $(13)$, установим соотношение $\Delta y_{0}+\left|y_{0}\right|^{\rho} y_{0}$ $+u_{0}=0$.

В силу вьпуклости и замкнутости множества $U_{0}$ справедливо включение $u_{0} \in U_{0}$. На основании полученных результатов заключаем, что пара $\nu_{0}=\left(u_{0}, y_{0}\right)$ оказьвается допустимой. Тем самым справедливо равенство $A \nu_{0}=0$. Учитьвая условия $\nu_{\varepsilon} \rightarrow \nu_{0}$ в $V$ и $A \nu_{\varepsilon} \rightarrow 0$ слабо в $L_{q}(\Omega)$, установим непрерывность оператора $A: V \rightarrow W$ в точке $\nu_{0}$, где под $W$ понимается пространство $L_{q}(\Omega)$ со слабой топологией. Как уже отмечалось ранее, непрерьвность оператора $A$ в точке $\nu_{0}$ достаточна для того, чтобы последовательность $\left\{\nu_{\varepsilon}\right\}$ оказалась слабой минимизирующей для задачи 2 . Действительно, в процессе доказательства теоремы 4 было установлено соотношение (12). Отсюда в силу включения $\nu_{0} \in U$ следует, что значение $\nu_{0}$, будучи пределом последовательности $\left\{\nu_{\varepsilon}\right\}$, оказьвается еще и решением задачи 2. Теорема доказана.

Итак, мы имеем алгоритм решения задачи 1 для сингулярного эллиптического уравнения. В его основе лежит регуляризованная задача 2 , для которой получены необходимые условия оптимальности (4)-(6). Отсюда можно определить ослабленное приближенное решение $\nu_{\varepsilon}$ при малых значениях параметра регуляризации. При выполнении неравенства $\rho<r-1$ это значение оказьвается достаточно близким в соответствующей топологии к решению задачи 1. 
5. Замечания. 1. Второе слагаемое в определении регуляризованного функционала напоминает стабилизатор в методе Тихонова [10]. Однако в последнем дополнительное слагаемое в регуляризованном функционале обычно выражает норму управления, а не состояния. Кроме того, метод Тихонова ориентирован на преодоление трудностей, обусловленных некорректностью экстремальной задачи. В данном случае соответствующий член вводится исключительно с целью получения дополнительной априорной оценки при доказательстве разрешимости регуляризованной задачи.

2. При $\varepsilon=0$ уравнение (6) совпадает с исходньм уравнением состояния (1). В этом и состоит смысл метода штрафа, согласно которому при неограниченном убывании параметра регуляризации, в принципе, обеспечивается выполнение уравнения состояния. Соотношения (4)-(6) при $\varepsilon=0$ приобретают форму необходимых условий оптимальности для исходной задачи. Однако получение соответствуюших предельных соотношений в общем случае подобно тому, как это делает Ж.-Л. Лионс при $\rho=2, q=2, r=6$ (см. $[1$, с. 265$])$, не представляется возможным. Проблема здесь не только в том, что имеющегося запаса функциональных свойств явно не хватает для обоснования предельного перехода в выражениях (4)-(6). В случае неразрешимости задачи 1 необходимые условия оптимальности для нее вообше не имеют смысла.

3. В теоремах 4 и 5 устанавливаются свойства не самой последовательности $\left\{\nu_{\varepsilon}\right\}$, а лишь ее подпоследовательности. Однако если бы существовала такая подпоследовательность из $\left\{\nu_{\varepsilon}\right\}$, которая не была бы минимизирующей в указанном смысле, то с помощью описанной процедуры из нее можно было бы извлечь подпоследовательность, обладающую всеми необходимыми свойствами. Не исключается также случай неоднозначной разрешимости задачи 1. Возможно, последовательность $\left\{\nu_{\varepsilon}\right\}$ имеет несколько предельных точек, оказывающихся решениями задачи. Тем не менее, обрьвая последовательность $\left\{\nu_{\varepsilon}\right\}$, мы все равно получим приближенное в указанном смысле решение задачи. Однако остается не известным, всякое ли решение может быть аппроксимировано указанным способом.

4. Поскольку приведенные выше результаты позволяют в определенном смысле исследовать экстремальную задачу даже при ее возможной неразрешимости, они вписьваются в общую концепци методов расширения экстремальных задач (см., например, [6], [9]). Однако в отличие от известных методов расширения здесь не просто осуществляется переход от исходной экстремальной задачи к заведомо разрешимой расширенной так, что нижняя грань исходного функционала совпадает с минимумом функционала в расширенной задаче. Решающим моментом здесь является принципиальньй переход от поиска, возможно, отсутствующего оптимального управления к явному построению заведомо сушествующей минимизирующей последовательности.

5. Полученные результаты не связаны со специфическими особенностями уравнения и могут быть распространены на другие сингулярные системы, в частности, на другие уравнения, рассмотренные в [1].

\section{СПИСОК ЦИТИРОВАННОЙ ЛИТЕРАТУРЫ}

[1] Лионс ЖК.-Л. Управление сингулярными распределенньми системами. М.: Наука, 1987.

[2] Фурсиков А. В. Свойства решений некоторых экстремальных задач, связанных с уравнениями Навье-Стокса // Матем. сб. 1982. Т. 118. № 3. С. 323-349. 
[3] Фурсиков А. В. Оптимальное управление распределенными системами. Теория и приложения. Новосибирск: Научная книга, 1999.

[4] Tiba J. Optimal control for second order semilinear hyperbolic equations // Control Theory and Advanced Technologies. 1987. V. 3. №1. P. 33-43.

[5] Сумин В.И. О достаточных условиях устойчивости существования глобальных решений управляемых краевых задач // Дифференц. уравнения. 1990. Т. 26. № 12. С. 2097-2109.

[6] Янг Л. Лекции по вариационному исчислению и теории оптимального управления. М.: Мир, 1974.

[7] Райтум У.Е. Задачи оптимального управления для эллиптических уравнений. Рига: Зинатне, 1989.

[8] Сумин В.И. О расширении оптимизационных задач, связанных с функциональными уравнениями в пространствах существенно ограниченных функций // Матем. модел. и оптим. управл. 1998. № 1. C. 126-133.

[9] Экланд И., Темам Р. Выпукльй анализ и вариационные проблемы. М.: Мир, 1978.

[10] Васильев Ф. П. Методы решения экстремальных задач. М.: Наука, 1981.

[11] Zolezzi T. A characterizations of well-posed optimal control systems // SIAM J. Control and Optim. 1981. V. 19. № 5. P. 604-616.

[12] Иоффе А. Д., Тихомиров В. М. Теория экстремальных задач. М.: Наука, 1974.

[13] Габасов Р., Кириллова Ф. М. Особые оптимальные управления. М.: Наука, 1973.

[14] Кларк Ф. Оптимизация и негладкий анализ. М.: Наука, 1988.

[15] Серовайский С. Я. Оптимальное управление для уравнений параболического типа с негладкой нелинейностью // Матем. ж. 2002. Т. 2. №1. С. 76-83; № 2. С. 87-94.

[16] Серовайский С.Я. Контрпримеры в теории оптимального управления. Алматы: Казак университеті, 2001.

[17] Лионс ЖК.-Л. Некоторые методы решения нелинейных краевых задач. М.: Мир, 1972.

[18] Гаевский X., Грегер К., Захариас К. Нелинейные операторные уравнения и операторные дифференциальные уравнения. М.: Мир, 1978.

[19] Ладыженская О. А., Уральцева Н. Н. Линейные и квазилинейные уравнения эллиптического типа. М.: Наука, 1964.

[20] Лионс ЖК.-Л. Оптимальное управление системами, описываемыми уравнениями с частными производными. М.: Мир, 1972.

[21] Ekeland I. The $\varepsilon$-variational principle revisited // Lecture Notes in Math. 1990. V. 1446. P. 1-15.

[22] Авербух В.И., Смолянов О. Г. Теория дифференцирования в линейных топологических пространствах // УМН. 1967. Т. 22. №6. С. 201-260. 